\title{
Towards an Ethic of Robotics
}

\author{
André Boyer \\ IPAG Business School \\ University of Nice-Sophia Antipolis
}

\author{
Faranak Farzaneh \\ IPAG Business School
}

\begin{abstract}
Since their appearance in the middle of the twentieth century in the industrial field, robots are developing in many economic sectors, around the use of artificial intelligence (AI). In this paper, after examining what the concept of robot means today, we analyze the ethical issues that robotics engenders in various sectors of society, starting with industry, through military and police robotics, health, education, household robots and ending with agriculture and the environment. From this analysis, we seek an ethical framework for robotics, which we try to define a problematic from the couple "action and responsibility" of robots.
\end{abstract}

Keywords: ethics, robot, robotics, artificial intelligence (AI), responsibility

\section{INTRODUCTION}

Robots have appeared in a generalised way in the industrial field since the first half of the 20th century and are developing rapidly today, both qualitatively and quantitatively, around the increasingly thorough use of artificial intelligence (AI) technologies. The latter provides robots with the ability to perceive, use language, interact with humans and the environment, solve problems, learn and even be creative.

As a result, robots with more or less AI can make decisions that are unpredictable for humans ${ }^{1}$, as they depend on the experience gained by robots and the stochastic conditions embedded in the AI. The question therefore arises, at the ethical level, of the responsibility for the actions performed by robots equipped with AI, the cognitive robots.

In doing so, we place this article in the field of management and organisation sciences, in order to introduce a new problem generated by the appearance of robotics, not only at work but in the whole field of human activities.

By "dividing" this problem into specific fields of research, for example on the future of work or on the management of an organisation impregnated by AI, it is a question here of taking the measure of the promises and risks of robotisation with regard to ethics as a whole, and in particular around the question of the responsibility of the actor, whether he is embodied by the company or by the individual. In fine, how to integrate the responsibility of a robot in the field of ethics, and especially in that of the ethics of otherness?

In an even broader perspective, robotisation can appear as the instrument of a transhumanism, or even an anti-humanism, which would modify not only work but all the living conditions of humanity. If this article does not pretend to address all these issues, at least it wishes to consider the possible extensions. 
We will begin by observing how the introduction of cognitive robots into human society has an impact on human behaviour, whether it induces social or cultural changes, which gives rise to the ethical questions that we examine in this article, relying first of all on the notion of the robot (1.) which will then enable us to describe the effects of the introduction of robots in social relationships (2.), from which we will examine what an ethics of robotics can mean (3.).

\section{The Notion of Robot}

The very term "robot" is on the borderline between the technical and the imaginary. Derived from the term robota, which means "work" in Czech, it replaces the term "automaton", appearing in 1920 in a work of fiction ${ }^{2}$. Since then, robots have been constantly mobilising the human imagination with a negative bias generated by the fear that they will supplant human beings.

\section{The Definition of the Term "Robot"}

We have defined a robot in its anthropomorphic sense (Farzaneh \& Boyer 2018) as a machine that provides services for human beings, either by substituting for them or by collaborating with them. Other definitions have been proposed by Rosenberg ${ }^{3}$ (1986) or by Angelo $^{4}$ (2007), but the evolution of robots must be taken into account in order to grasp the evolving meaning of the concept of robot. Thus Gibilisco (2003) distinguishes four generations of robots, each one acquiring increasing capacities compared to the previous one:

- Before 1980, the first generation of robots were based on stationary servomechanisms and used neither ex-tern sensors nor artificial intelligence.

- Between 1980 and 1990, second-generation robots were programmable, controlled by microprocessors and equipped with visual and tactile sensors.

- From the 1990s onwards, third generation robots became mobile and autonomous, capable of recognising and synthesising speech and integrating artificial intelligence and navigation systems.

Finally, Gibilesco predicts that future generations of robots should be able to gradually acquire most of the characteristics of the human brain, except those that are purely biological in nature.

However, since this classification, robotics has diversified beyond mechanical and electrical engineering, which deals specifically with robots or "bots", to include nanosciences for "nanobots", biology for "biorobots" or "cyborgs", and even botany with "plantoids", whereas it would seem that the ethics of robotics has remained confined to robots already in use and not to the robots of the future.

As a result, there is a risk that this ethic will constantly lag behind the emergence of new types of robots. Moreover, robotics is not only a matter of science but also of human imagination, hence the risk of an anthropomorphic bias towards "real" robots.

\section{The Imagination of Robots}

The human mind is haunted by the demiurgic temptation to create intelligent beings, which is why such beings are represented in mythology, literature or cinema. We shall quote, for example, to limit ourselves to recent representations of robots:

- Mary Shelley's Frankenstein (1818), which describes an artificial living being who revolts against his creator in the name of his right to happiness.

- The play by Karel Capek, R.U.R. or Rossum's Universal Robots published in 1920, which, as mentioned above, created the term "robot". The theme of the play reflects the social concerns about robots. The latter, made of organic matter, are intended by the inventor to serve as cheap labour power, but they revolt against their creators and seize power.

- Isaac Asimov introduces the term "robotics" and proposes in one of his short stories, Vicious Circle, (1942) an ethics of robotics based on three laws:

- a robot cannot harm a human being, nor, by remaining passive, allow a human being to be exposed to danger; 
- a robot must obey orders given to it by a human being, unless such orders conflict with the first law;

- a robot must protect its existence as long as this protection does not conflict with the first or second law.

Asimov then judged that these three laws should be supplemented by a Zeroth law which he proposed in The Robots and the Empire (1985):

- A robot cannot harm humanity, nor, by remaining passive, allow humanity to be exposed to danger.

Previous works translate, through revolt (Frankestein), the use of robots against human labour (U.R.) or the need to control the behaviour of robots (Vicious Circle), the fear of a man overwhelmed by the creatures he has designed and made. This fear has been widely expressed in science fiction films and series, such as, to mention only the most famous works, in Fritz Lang's Metropolis (1927), which describes the revolt of robots against human beings in 2001, Stanley Kubrick's Space Odyssey (1968), which presents a computer controlling all the functions of a spaceship and seeking to destroy its human crew, or James Cameron's Terminator (1984), which describes a future world constituted as a dictatorship governed by artificial intelligence and an army of robots.

However, this presentation of robots is not systematically catastrophic. The duo of robots R2-D2 and C3PO in Star Wars by George Lucas (1977) has human qualities; similarly, the child robot David in A.I. Artificial Intelligence by Steven Spielberg (2001) suffers from having been abandoned by his biological mother.

These approaches to the imagined behaviour of robots have religious foundations. Indeed, Christianity and Judaism consider the creation of such beings as an illegitimate interference with the role of the Creator, whereas Buddhism and Shintoism, on the contrary, consider all living and non-living beings as endowed with a soul or a spirit. The latter approach is reflected in Japanese culture by an acceptance of humanoid robots (BarCohen and Hanson, 2009).

It should be noted that these representations of robot behaviour largely preceded the practical arrival of robots in human society, but it should also be remembered that these representations influence the ethical concerns of humanity with regard to robots. The first industrial robot (Stone, 2005), Unimate, was only designed in $1954^{5}$ and the first AI-based robot was developed between 1966 and $1972^{6}$. We can identify the characteristics of existing or planned robots from an ethical point of view by only referring to the current state of robotics and by trying to extend the trends to imagine the state of robotics in a few decades at the most.

Robot Capabilities and Ethical Issues

A number of characteristics of robots, such as mobility, interactivity, communication and autonomy raise ethical questions.

- The mobility of robots: while most industrial robots are not mobile, the activity of other types of robots requires it. They can then generate risks for human beings, all the more so as they have a high degree of autonomy and advanced capacities for interaction with their environment.

- Interactivity with the environment distinguishes robots from computers, by equipping the former with sensors such as cameras or lasers or actuators such as arms or grippers. The ethical question is directly linked to this capacity for action of robots, which can inflict damage on human beings in the event of malfunction.

Moreover, through their sensors, robots can collect data that can harm human beings, in the form of espionage or other criminal activities.

- Communication with robots now takes place directly with humans using speech recognition and text-to-speech systems. The question therefore arises as to the level of comprehension of human languages by robots. Indeed, human languages have multiple meanings with complex contextual aspects and implicit presuppositions (Wise, 2005) whereas robots need explicit instructions ${ }^{7}$. 
- Autonomy is substantial to the robot, which "essentially holds a certain degree of autonomy or the ability to 'think' alone and make its own decisions to act on the environment" (Bekey, 2012, p.18). Unlike early robots that were only capable of performing repetitive tasks, today's robots can perform complicated tasks without relying on commands or direct human control. The question therefore arises as to the level of performance that an autonomous robot must achieve compared to that of a human being performing an identical task. There is also the question of man-machine cooperation (Brynjolfsson and McAfee 2011), since it would seem that the performance of the man-machine pair is superior to both men and machines working alone.

\section{The Extension of the Notion of Robot}

If, in the human mind, the notion of robot merges with that of machine, it must now be extended to nanorobotics, the purpose of which is to create and develop robots whose components are a few nanometres in size, called nanobots, nanoïds, nanites or nanomites.

In addition to this reduced dimension, materials are used which can be organic with specific capacities ${ }^{8}$ (Ummat et al., 2004) as well as inorganic (Weir et al., 2005). Invisible to the human eye, nanorobots require consideration of the level of security and privacy required to protect humans and ecosystems.

\section{AI and the Paradigm Shift in Robotics}

While most industrial robots do not require AI, since their behaviour is pre-programmed to automatically perform a limited number of specific and repetitive tasks in a fixed environment, robots with AI are able to perceive and formally represent changes in their environment and adapt their operation accordingly.

Alan Turing (1950) offered the first theoretical formulation of a possibility of artificial intelligence (AI) by proposing a test to decide whether intelligence can be attributed to a robot when a human being, placed in an experimental context, proves unable to distinguish whether the answers he obtains come from the robot or from a human being. Although to date this test has never been validated by a robot (Franklin, 2014), there are now robots capable of solving problems in a way similar, if not identical, to human thought. It is then assumed by definition that such a robot is equipped with AI, which changes the paradigm that is now applied to robots.

Indeed, the first robots equipped with AI were still programmed on the basis of a hierarchical paradigm, whereas the most recent ${ }^{9}$ are based on a reactive paradigm. However, applying a hierarchical paradigm to a robot means that it is supposed to start by capturing the necessary information before preparing its action.

It is therefore necessary, beforehand, to define in which framework the robot is supposed to act, which implies defining in advance which information is relevant and irrelevant for its action, leading to an almost unlimited collection of data.

This practical difficulty has led to the use of a reactive paradigm, inspired by cognitive psychology. Action planning is limited, which frees computing power, and the robot's actions are linked to sensory data.

However, regardless of the paradigm used, robots perform their tasks on the basis of algorithms that control both the predictable behaviour of deterministic robots and the learning capabilities of AI-enabled cognitive robots.

Simply, the behaviour of a deterministic robot is pre-programmed, which solves the traceability of its actions when it comes to resolving an ethical or legal dispute. On the other hand, cognitive robots, based on AI, learn from their experiences to recalibrate their algorithms themselves. It is therefore no longer possible to completely predict behaviours, which creates ambiguity about the respective responsibilities of the designer and the user, not to mention that of the AI robot itself.

This is why the ethical issues related to robotics must integrate the rapidly evolving characteristics of robots in the framework of their social-economic relations with human society.

\section{Ethical Effects of Robotisation in Social-Economic Relations}

Depending on the speed of its development and the importance of its effects on the ethics of the sector concerned, we have chosen to deal successively with industrial robots which historically constitute the first 
field of robotics development, military and police robots which raise fundamental questions in terms of ethics, transport robots, robots in the health sector, the education sector, household robots and robots in agriculture and the environment which constitute the most advanced fields in terms of robotics development.

\section{Ethical Effects of Industrial Robotics}

The first involvement of robots in social relations can be seen in the progressive replacement of workers by robots in industry. Since the middle of the 20th century, industrial robots have gradually replaced human operators for repetitive tasks in the manufacturing sector, while newer robots, equipped with AI, are beginning to perform manual tasks of a non-repetitive type. As the cost of robots declines and their technological capabilities increase, they are gradually replacing human labour in a wide range of service sector functions (Frey \& Osborne, 2013). This leads to increased productivity but also, initially, to higher unemployment. In this respect, we can point to the emergence of a new period in history that would be characterized by the "end of work" (Rifkin, 1995), even if there is not enough hindsight to assess all the consequences of a strong increase in the use of robots on human activity and particularly on its social effects. However, two trends seem to emerge regarding the effects of robotics on the distribution of power in general and on working conditions in particular:

- Robotics is characterised by a convergence of technologies, rapid progress and a progressive reduction in costs which must be integrated into our societies while at the same time modifying them. First of all, the impact of robotisation on the globalised economy is asymmetrical. Increasingly cheaper and more efficient robots are gradually pushing out of the labour market, unless the energy and raw material costs of robots are taken into account (Farzaneh \& Boyer 2018). This effect manifests itself both in the national labour market and through globalized competition between low and high wage cost countries.

Low-paid workers will find it difficult to compete with the productivity of robots. In this respect, there is a shift of production from highly automated plants to plants close to their markets, which better addresses the costs and risks, both financial and environmental, of a long supply chain (Balding, 2016). The risk is therefore that the development of robotics will widen a technological, social and political gap between and within societies, by changing power relations. However, it is already perceptible that it is modifying social exchanges (Peláez, 2014).

- The thesis of the end of the work, with its procession of social impacts integrated in the paradigm of a post-modernity succeeding Fordism (Durand, Boyer, 2000) may be reinforced by the appearance of robotics. However, the relationship between postmodernity and its relation to work, and more generally to meaning and ethics, has been addressed in the work of Bruna, Peretti \& Yanat (2016) ${ }^{10}$, completed by Bruna, Montargot \& Peretti (2017), which shows how diversity at work is part of a sustainable development perspective, The links between the concept of diversity, the 'totem' of the norm and the ideology of control affecting the postmodern enterprise are clarified in Bruna, Ducray \& Montargot (2017).

- Indeed, advances in robotisation are profoundly changing working conditions and jobs. Working side by side with robots requires new professional skills and the implementation of new and adequate safety measures in the workplace. Three categories of robots are present in workplaces where they affect safety, industrial robots, service robots and professional and collaborating robots:

- Most industrial robots are not aware of their environment, which makes them dangerous for workers, and it is therefore important to maintain a sufficient distance between human workers and active robots by creating reserved spaces.

- Service robots are mainly used outside industrial sites, in unstructured and unpredictable environments. They generally cannot be isolated from workers, as they often share the same workplace and the complexity of the environment means that robots must be given a certain degree of autonomy and mobility, which can create dangerous situations for workers. 
- Collaborating robots are designed for direct interaction with a human being; they include both industrial robots and professional or personal service robots, combining dexterity, flexibility and the ability to solve problems with the strength, endurance and precision of mechanical robots. As collaborating robots work side by side with human work, safety cannot be achieved by isolating the robot, which requires other methods.

\section{Ethical Effects of Police and Military Robotics}

The aim is to examine the consequences of the use of robotic systems in armed conflict with regard to the application of the most important principles of International Humanitarian Law (IHL), namely the principles of distinction, proportionality and responsibility. The following is a discussion of autonomous police surveillance, drones, autonomous weapons and the hijacking of robots:

Robotics is increasingly applied to surveillance. Like video surveillance and satellites, drones can see and hear continuously. They can, for example, be used to control borders, crowds or traffic. This has major implications for privacy and data protection. In this area, the adaptation of military technologies can lead to a logic of management of society based on an economy of fear, that of 'an omnipresent enemy' (Crandall and Armitage, 2005, p.20). In particular, the use of robots equipped with so-called 'non-lethal' weapons in the repression of forms of public protest can be used as a tool of oppression, as the robots do not risk disobeying an authoritarian regime in the same way as soldiers or policemen might be led to do.

- An Unmanned Aerial Vehicle (UAV) is defined as "a land, sea or air vehicle that is remotely or automatically controlled" (Chamayou, 2015, p.11). They open up new possibilities on the military level while creating new risks on the moral level ${ }^{11}$. However, distinguishing a combatant from a civilian in a remote-controlled situation is inherently difficult and the greatly increased complexity of the data series on which firing decisions are based increases the risk of error in target identification. Furthermore, assessing remote proportionality is problematic, as the UAV pilot must, individually or with his team of analysts, decide to apply lethal force on the basis of contextual data that is often insufficient ${ }^{12}$. As far as liability is concerned, the aim is to verify that UAVs are used by regular military forces for the same purposes as those used for conventional aircraft.

- More generally, autonomous weapons are weapons that, once activated, select and attack targets without additional human intervention. They raise the same ethical questions, in terms of the principles of distinction, proportionality, accountability and transparency, but in this case the absence of human intervention raises concerns about the ability of software to make decisions that would normally be made by a human being, in particular because life and death decisions can hardly be made by machines, ${ }^{13}$ whereas the power to kill or to delegate the power to kill ${ }^{14}$ cannot be attributed to a machine without denying what constitutes the core of an individual's moral being and its intrinsic value, human dignity. Moreover, robotic weapons have strategic implications, in particular by lowering the threshold for triggering armed conflict ${ }^{15}$ and pose problems in terms of security, for example in terms of hijacking or piracy.

- Finally, the market for small unmanned vehicles raises ethical questions in terms of respect for privacy, because of the risks of criminal use of these products ${ }^{16}$.

\section{Ethical Effects of Transport Robotisation}

The transport sector is one of the areas where robotics is most used and accepted because the automotive industry is increasingly organised around automation. With the evolution of sensors, an ever-increasing proportion of driving functions are delegated to robots, such as speed control, parking or lane changing. A qualitative leap forward is being made with the emerging technology of autonomous vehicles (AV), which will not only assist the driver, but also replace him completely by relying on cognitive algorithms.

The ethical problems posed by AV technology lie in the opposition between individual and collective interest. While from the first point of view, once the technical difficulties have been overcome, AV offers many advantages, particularly in terms of safety, the fact remains that decision-making on machines on the basis of moral principles must ensure that either individual interest or the public good prevails. For example, 
in the event of an unavoidable accident, should priority be given to minimising the number of victims, even if this means sacrificing the vehicle's occupants, or to protecting passengers at all costs ${ }^{17}$ ? Finally, the question is whether these issues should be regulated at national or international level, whether they should be subject to standards or codes of conduct for autonomous vehicles or left to market forces.

\section{Ethical Effects of the Use of Robots in the Health Field}

We will successively examine the ethical issues generated by the use of medical robots, nursing robots, care robots for the elderly and companion robots.

- Semi-autonomous robots are used in surgery and have many advantages ${ }^{18}$, but surgeons express some technical reservations about robotic surgery, such as the loss of the sense of touch. However, surgical robotics already raises an economic and ultimately ethical question, as the multiplication of robots in surgery has implications for the allocation of resources in public health systems, due to the fact that it is currently more expensive than an operation with a normal surgeon.

Nursing robots are used in some therapeutic approaches with autistic children and children with Down syndrome. However, the few studies devoted to the use of robots in this context do not indicate that the autistic robot-child dyad is functionally superior to the autistic parent-child dyad (Simut et al., 2016). Similarly, exoskeletons used to help people with disabilities to increase their mobility are part of the development of medical robotics, but, like neurological implants or nanorobots, they open the way to a transformation of the human body and the question arises, from an ethical point of view, of how far the development of hybridisation should go and for what purposes.

Robots for elderly care are part of social robots, which are robots that communicate and interact with human beings. The notion of companion robots is also used to highlight the functional and emotional dimensions of this category of robots (Oost and Reed, 2010). However, due to the rapid ageing of the population and the difficulties in recruiting care personnel, robots appear as a means of bridging the gap between the need for and the supply of care services. Thus, robots providing physical assistance (cleaning, cooking, personal hygiene), companion robots (stimulation of cognitive activities) and health and safety monitoring robots (risk of falls, heart failure, mobility disorders) are appearing on the medical market. These robots raise the question of the means used and the ends sought. For example, how can behavioural control and autonomy of older people be balanced with the use of robots? Is the aim to improve the quality of life of older people or to reduce the work of care staff or, more generally, to relieve society of the burden of caring for older people (Wu et al., 2010)? Depending on the objective chosen for these nursing robots, the questions of their cost, care efficiency or acceptability ${ }^{19}$ to older people become more or less important. Finally, companion robots are being developed in the field of sexuality and pose the question of the reduction of sexuality to the individual and not to the couple, questioning interpersonal relationships.

\section{Ethical Effects of the Use of Educational Robots}

With the rapid development of communication technologies, more and more multimedia tools are being used in education, including now educational robots.

Educational robotics enables the exploration, design, modelling, programming, construction and testing of learning concepts, based on individual or collaborative learning activities. From an educational design perspective, the use of robots is part of a constructivist model of learning that involves the active participation of students in the construction and acquisition of knowledge. It is a participation that is not necessarily acquired, since on the one hand, interest in robots may prove to be ephemeral for learners and on the other hand, because the introduction of robots into the system depends on cultural values, which make Japanese or Korean culture seem more suitable (Tzafestas, 2016) than European culture, for example (EC, 2012).

From the point of view of the ethics of the individual, one of the main ethical questions arising in this context concerns the role assigned to the robot and in particular to what extent it can replace a teacher. Another important issue concerns the relationship between robots and children, who are more likely than adults to attribute cognitive, behavioural and emotional characteristics to robots (Beran et al., 2011). 


\section{Ethical Effects of the Use of Household Robots}

The purpose of household robots, also known as service robots or domestic robots, is to help human beings perform certain tasks considered to be arduous or to serve as a means of entertainment ${ }^{20}$.

From an ethical point of view, the first risk concerns the damage they may cause, resulting from inappropriate force or speed, misunderstanding the instructions for use or error in the performance of specific tasks. The consequences may be aggravated when the users are children or the elderly. In addition, they can easily be diverted from their official use for unethical purposes. Often equipped with cameras and microphones, they can easily be turned into tools for intruding into private life and they can store a lot of private and confidential data (photos of the interior of a house and its inhabitants, data on their habits, passwords of their alarm system, location of their precious objects). The danger is even greater when household robots are connected to the Internet or another network that is insufficiently protected and easy to penetrate. In such situations, hacking the robot can facilitate criminal activities such as theft or blackmail.

The outward appearance of robotic toys and companion robots is also an ethically sensitive issue (Pearson and Borenstein, 2014). Their shape and appearance should reflect the collective aesthetic preferences of a culture, not reinforce gender stereotypes, be developmentally appropriate for children and pay particular attention to the degree of human appearance of such toys, as the positive or negative impact of such appearance generally varies according to the age or personality traits of their users.

\section{Ethical Effects of the Use of Robots in Agriculture and the Environment}

Robots are present in agriculture, for example in the dairy sector where cows can be milked by a robot, with their feed adapted according to the level of production and the age of the animal to maximize their productivity. In this particular case, from an ethical point of view, the transformation of the relationship between animal and man must be taken into account, as cows lose all familiarity with human beings. From the same ethical point of view, the question of animal welfare in an animal-machine model of productivity arises (Driessen and Heutinck, 2015).

UAVs are also present in agriculture, for which they provide data for more efficient use of chemical inputs or irrigation, thus contributing to sustainable agriculture, or for the selection of more resistant plants, thus increasing agricultural productivity. All in all, they are part of a model of intensive and precision agriculture, which induces a change in the relationship of human beings to the land (Leopold, 1949), which also raises an ethical question.

As such, the usefulness of robots for environmental protection, in oceanic research, in space exploration or in the dramatic case of monitoring and restoring the environment after a nuclear or chemical accident (Lin, 2012) must be weighed against the environmental impact of the entire robot production cycle. This cycle includes the extraction of rare earth elements and other raw materials, the energy needed to produce and power the machines and the waste generated during the production of robots and at the end of their life cycle.

It is likely that robotics will reinforce concerns about the growing volume of electronic waste and the depletion of earth element resources caused by the computer industry (Farzaneh and Boyer, 2018; Alonso et al., 2012) and it does not seem that in-depth studies on the environmental impact or ecological footprint of robots, and consequently the ethical issues generated by this negative impact, have already been carried out.

By examining the effects of the introduction of robots in an increasing number of areas of human professional and personal life, we have been able to observe the extent and variety of these effects on the organisation of human society; these effects may ultimately lead to profound changes in man's perception of the meaning of his environment. Ethics looks at the judgements of man or society on this perception of the environment transformed by robots.

Its foundations being renewed by the irruption of robotics in the organization of human society, we seek hereafter to set the framework. 


\section{In Search of an Ethics of Robotics}

As the ethical framework of robotics (3.1.) is under construction, insofar as it follows rather than accompanies the rapid changes of its developments, we will try to contribute to this construction through an analysis of the problematic of robotics (3.2.) which leads us to the question, central from an ethical point of view, of the attribution of responsibility for the actions of robots (3.3.) to lead to a reflection on the concept of the capacity for action of robots (3.4.).

\section{In Search of an Ethical Framework for Robotics}

Proposals to define such a framework are still rare, but two of them emerge from two different levels of analysis of the ethics of robotics, those of Ingram et al (2010) and Riek and Howard (2014). The former focus their recommendations on the behaviour of roboticist, while the latter define obligations not only for roboticists but also for all those involved in the design, manufacturing and marketing of robots.

For Ingram et al (2010) first of all, a roboticist must be concerned about the well-being of all human communities among which they distinguish roboticists, customers, users and employers. Moreover, a roboticist must, as much as possible, foresee possible unethical uses of his creations and try to limit them. They add in their draft code of ethics the respect of the physical well-being and rights of individuals as well as the rules to be considered to prevent inaccurate information and conflicts of interest. Naturally, it remains to translate these general recommendations into precise rules and ultimately into action. This is why the robotics professions have developed consistent codes of conduct, such as the IEEE (Institute of Electrical and Elecronics Engineers) code of ethics or the ACM (Association for Computing Machinery) code of ethics, but these ethical codes, specifically designed for robotic engineers, are still in their infancy and it is not yet possible to observe the practical consequences of their application.

Riek and Howard (2014) address a wider audience than roboticists. They propose a "code of ethics for human-robot interaction professionals", which contains a "primordial directive". The latter recommends that research, development and marketing activities concerning human-robot interaction should be based on the general principle of respect for the human person as well as on other principles, such as respect for human emotional needs, the right to privacy, the existence of circuit breakers and the need to limit the humanoid aspects of robot morphology.

These two attempts to define an ethical framework for robotics have not led, in spite of the initiatives we have mentioned, to professional ethical codes and ethical guidelines on how to implement robotics projects. No doubt such guidelines should emerge from the initiatives taken to promote an ethical regulation of robotics:

- Concerning the ethics of robotics research, Allistene (2016), from a consortium of French research institutes including CNRS, CEA, INRA, CDEFI, among others, has made several proposals to strengthen the ethical responsibility of robotics researchers regarding human-robot interaction.

- The Draft Report containing recommendations to the Commission on civil law rules on robotics, published in 2016 by the Legal Affairs Committee of the European Parliament, proposes an ethical framework for robotics and the robot industry. The report addresses data protection and privacy, the damage that could be caused by the new generation of robots and the liability of robot manufacturers, the precautionary principle, testing of robots in real environments, informed consent in robotics research involving human beings, disruptive devices (circuit breakers) and the impact of robotics on employment and education.

It also stresses the importance of traceability and the implementation of an advanced robot registration system. (JURI, 2016, p.13).

- The report of the World Commission on the Ethics of Scientific Knowledge and Technology (COMEST, 2017) synthesizes the different issues of robotics ethics and proposes a series of recommendations for an ethical code.

However, many contributions are proposed today on the ethics and law of robotics (Asaro, 2012; Calo, 2015; Holder et. al., 2016; Leenes et. al., 2017; Matsuzaki and Lindemann, 2015), while at the practical level robotics remains largely unregulated. Currently, potential damage caused by robots is covered by civil 
legislation on product liability, while the increasing autonomy of robots blurs the line between the responsibilities of robot manufacturers and users (Asaro, 2012).

Moreover, robot producers warn against the risk of slowing down the development of robots through a priori standards that would increase their level of uncertainty, as it appears that regulation of robotics must remain in line with the rapid evolution of technologies, while seeking a balance between the protection of fundamental human rights and values and the preservation of innovation (Leenes et al., 2017). However, the debate on the regulation of the issue cannot be settled without first settling the debate on the foundations of the ethics of robotics.

\section{Towards a Problematic of the Ethics of Robotics}

This problematic is based on the technological revolution brought about by robots, which, from an ethical point of view, blur the boundaries between human subjects and objects.

This blurring is evident when robots show a certain ability to make decisions, to express feelings, which allows them to take on an increasing number of human tasks. In doing so, they modify ethical notions such as capacity to act and responsibility, when they do not blur the values that are expressed in different areas of life. This blurring also induces a debate within society that oscillates between what we can call technooptimism, expressed by the utopia of transhumanism, and a techno-pessimism that invokes the need for bioconservatism to defend humanity against the overly radical impact of technologies.

Techno-optimism maintains that technological progress cannot be slowed down and must therefore be used to best advantage ${ }^{21}$. If we idealise the effects of technological progress, then we join the transhumanist ideal of using technology to improve human capabilities (Hottois et al., 2015). Techno-pessimism is concerned that technological innovations will affect social relations and the cognitive capacities of humanity. This is why it advocates a bio-conservatism around human dignity (Fukuyama, 2002) or the ability to be the author of one's own life (Habermas, 2003). If it is logical that robotics, as an emerging techno-social phenomenon, generates new ethical stakes, it is also necessary, beyond the global choices generated by techno optimism or pessimism, to deepen the concrete questions that robots raise on a practical level in order to draw the limits of the ethical debate. Thus, the first question that emerges, at the ethical level, is that of establishing the acts of a robot, in order to be able to appreciate them.

\section{Multi-Responsibility With Regard to Robots}

The question raised is who exactly is ethically and therefore legally responsible when a robot causes damage to human beings, property or the environment ${ }^{22}$. This requires traceability, which refers to the need to be able to determine the causes of a robot's actions (Riek and Howard, 2014, p.6). However, the requirement of traceability is not compatible with the development of robots with a high degree of autonomy, decision-making capacities and learning skills, because robotics, by pursuing "two contradictory tasks: increasing the autonomy of robots and, at the same time, ensuring that they are risk-free" (Matsuzaki and Lindemann, 2016, p.502), calls into question the notion of traceability. The responsibility for a robot's action appears to be most often shared between the designer, the engineer, the programmer, the manufacturer, the investor, the seller and the user of the robot, as none of these actors can be designated as the ultimate source of the action.

If none of the actors is entirely responsible for the act of a robot, then a potentially paralysing effect for robotics is manifested, hence the two ways proposed to solve the question of responsibility. The first would be to develop techniques to anticipate as far as possible the impacts of robotic development (Verbeek, 2013). The second would be to think carefully about the inevitable appearance of unforeseen effects, considering that the introduction of robotic technologies into society is a social experiment" (Van de Poel, 2013). However, the question now arises as to the nature of the action of robots

\section{The Ability of Robots to Act}

Robots can act autonomously by interacting with their environment on their own. However, on the one hand, the capacity to act has always been considered to be a human characteristic and the autonomy of robots remains relative. Indeed, it is the product of the work of designers and programmers which leads to 
learning processes of cognitive robotic systems. From the latter comes the action of robots which is the direct consequence of their own interactions and decision-making processes, but which is no longer the direct consequence of the instructions provided by their designers. This question of direct or indirect relationship between the design of a robot and its actions is the crux of the determination of the responsibility of the actors, between the robot, its users and finally its designers.

From an ethical perspective, an agent is responsible for his or her actions when he or she acts freely and intentionally, without being directed or coerced. It can therefore be concluded that robots do not have a freedom and intentionality comparable to that of human beings, but robots do have a certain degree of freedom since they can make decisions based on a certain form of intentionality linked to the algorithms that govern them.

To escape the alternative of describing robots either as technological objects or as quasi-human subjects, Rosenberger and Verbeek, (2015) propose the approach of technological mediation which presents robots as mediators ${ }^{23}$ between human beings and their environment. According to this approach, when a robot is introduced into a specific practice, such as teaching, nursing or cleaning, it plays a mediating role that modifies the action practice of human beings. It is then no longer a question of comparing robots to human beings, but of determining how robots modify human practices. Finally, the question arises of the status of robots as ethical agents and its implications in the field of management sciences.

\section{Management Sciences and the Ethical Status of Robots}

We have successively defined the notion of robot and its double extension, organic and with AI, its effects on social and economic relations by analysing them through the sectors where the action of robots seemed particularly significant, before looking for the foundations of an ethics of robotics for which the origin of the responsibility of robots seems to be determining. It is therefore a question of first considering the influence of robotisation on the ethics of the company before considering the construction of an ethical status for robots .

\section{Robotisation and the Ethics of the Organisation}

As robots develop more and more autonomously, society is forced to draw up rules to manage them, as these technologies, with their immense potential benefits, come with a large number of dangers that can be

As robots develop more and more autonomously, society is forced to draw up rules to manage them, as these technologies, with their immense potential benefits, come with a large number of dangers that can be taken into account by the principles of organisational ethics.

It should be remembered that many assembly lines jobs around the world have already replaced human workers with robots, thus improving safety at work, reducing the risk of errors and accidents at work and increasing productivity. From this point of view, therefore, robots may appear to be an ethical choice, but it should be noted at the outset that the use of robots threatens to eliminate part of the jobs, alienate humans from the meaning of work and leave people without any human contact ${ }^{24}$.

It is therefore legitimate to ask the question of the optimal use of robots and AI in the company. When it is possible to make a choice between robots and human beings, it is necessary, from an ethical point of view, to examine the advantages and disadvantages of the use of robots, in particular to determine the costs of the robotic choice and to define the boundaries to the use of robotics and, correlatively, $\mathrm{AI}^{25}$.

The introduction of robots and AI into the organisation has an influence on human rights. While AI can enable recruitment agencies to search for the best talent on the Internet by relying on social media profile checking of job applicants, while job seekers can send their CVs faster and cheaper to a large number of companies, ${ }^{26}$ this search for information has an impact on justice and equity and is leading to new regulations, such as the Canadian Personal Information Protection and Electronic Documents Act. (Personal Information Protection and Electronic Documents Act ou PIPEDA) ${ }^{27}$.

In addition, it is tempting to assign AI to surveillance activities that violate human rights based on satellite photos, facial recognition or publications on social networks, which is beginning to provoke protective reactions, such as the decision of the city of San Francisco, which since 14 May $2019^{28}$ has adopted an order banning the use of facial recognition technologies by the police and other municipal 
bodies. Before abuses are committed, the ordinance is preventive, to ensure that citizens can control how they are overlooked and monitored.

Based on a set of collective behaviours, a culture is thus established that makes it possible to protect the massive and ever-increasing volume of information collected on individuals and organisations (Dhillon, 1997, Lim et al., 2009). Corporate cultures are impacted because security breaches in IT systems are the result of human coding errors29 and employee behaviour has an impact on information security in organisations (Andersson et al., 2014).

Through different facets of robotics and artificial intelligence technologies, robotics and AI thus raise multiple ethical questions whether on the appropriateness of their use or on the control of the collected information, which lead us to consider the construction of an ethical status of robots.

\section{Ethical Status of Robots}

If the malfunctioning of robots can inflict damage on people, it is not just a question of asking roboticists to respect ethical standards, but of enshrining ethical standards in the programming of robots.

This could lead to the emergence of a new discipline that we will call Robotics Ethics, intended to "provide robots with ethical principles or a procedure for resolving the ethical problems they may encounter" (Anderson and Anderson 2011, p.1).

This raises the question of the possibility of constructing artificial moral agents, which was initiated by the proposal to distinguish implicit and explicit ethical agents (Moor, 2011). According to this conception, a robot is an implicit ethical agent insofar as it is equipped with software that prevents or restricts unethical behaviour ${ }^{30}$. However, for robots to become explicit ethical agents, they would have to be programmed to act according to principles and ethically justify their actions. However, the creation of such robots is not yet effective, even though current chess computer programs pave the way for this type of robot (Whitby, 2011).

For such robots to be programmed, an ethical code must first be chosen. Asimov (1950), in a work of fiction, has had the merit of proposing three laws of robotics that are not applicable in practice because they are too general, potentially contradictory, and obtrude the philosophical principles on which they implicitly rely. We are thus brought back to this level, to a debate on the choice of an ethics of robots based on a human ethic among several possibilities.

However, it remains to be seen, when robots will be endowed with explicit ethics in the future, what the ethical rights of robots will be. Finally, is it conceivable that robots will in future be entitled to protection against damage identical to that granted to human beings and certain animals?

To the extent that robots are capable of performing cognitive tasks on their own, they possess a limited form of rationality, without free will, intentionality or self-awareness. Moreover, they do not experience feelings, even though sociable robots can be programmed to develop artificial feelings. (Valverdu and Casacuberta, 2009).

However, the appearance in the future of human-machine or animal-machine hybrids or cyborgs, robots integrated into a biological organism or containing at least certain bio-logical components, could call into question this simple distinction between humans and robots. It is indeed the shifting of the frontier between man and object that was the starting point of our analysis, it is this shifting of the frontier that generates the need to develop an ethics of robotics, which remains to be defined and to evolve as the frontier moves. This is why we tried to show in this article the main data to be taken into account for the dynamic elaboration of an ethics of robotics, in particular around the concept of responsibility, in front of the present challenges of postmodernity and even of a transhumanist future.

\section{ACKNOWLEDGEMENT}

Another analysis of data was also published: Boyer, A. \& Farzaneh, F. (2019). Vers une éthique de la robotique: Towards an ethic of robotics. Question(s) de Management, 2(2), 67-84. https://doi.org/10.3917/qdm.192.0067

Translated \& edited by American Publishing Services (https://americanpublishingservices.com/). 


\section{ENDNOTES}

1. https://www.archyworldys.com/eric-sadin-artifi-cial-intelligence-breeds-a-progressive-banishment-of-thehuman/

2. The term "robot" was created by the Czech Karel Capek in a 1920 science fiction play called R.U.R. (Rossumovi Univerzální Roboti).

3. "Machine equipped with sensors or instruments for detecting input signals or environmental states, but also including reaction or orientation mechanisms and capable of performing detection, calculation or other tasks, and recorded programmes determining the sequence of actions."(Rosenberg, 1986, p.161).

4. "Intelligent machine capable of performing routine, repetitive or dangerous mechanical tasks or other operations directly at the command of a human being or autonomously, using a computer with embedded software (containing previously recorded commands and instructions) or based on an advanced level of (artificial) machine intelligence (which allows decisions and actions to be based on data collected by the robot in its current environment)". (Angelo, 2007, p.309).

5. By Joseph Engelberger and George Devol for the General Motors assembly line in Trenton, New Jersey.

6. Shakey, a wheeled robot designed by Charles Rosen and his associates at the Center for Artificial Intelligence in California.

7. This is why developers are offering increasingly sophisticated speech recognition and text-to-speech systems. For example, the humanoid robots Wakamaru (Mitsubishi) and Nao (Aldebaran Robotics) can communicate with human beings by both gesture and speech (Bekey, 2012).

8. In the case of organic nanorobots, they are natural molecular machines consisting of an assembly- of proteins with pre-programmed biological functions that can be activated in response to specific physicochemical stimuli in vitro or in an artificial medium. Organic nanorobots have specific capabilities, such as their durability, rapid activation, ability to extract energy, collective intelligence, ease of reproduction, and interface architecture from the nano to the macro level (Weir et al., 2005), with mainly medical applications.

9. This is how the Kismet robot, designed half a century after the Shakey robot, aims to achieve social interaction between the robot and human beings: it can move its eyes, change its facial expression according to its mood, communicate with human beings through speech and react to the emotions of its interlocutor.

10. The article questions two taboos, the "totemism" of the norm and that of immediacy, the first of which invites us to resolve the antinomy between a search for predictability and the need for innovation, and the second which questions the relationship to time as a lever for transforming mentalities and redesigning organisational systems and practices, with a view to designing a management system that makes it possible to achieve sustainable socio-economic performance.

11. The UAV operators are at a distance from the armed machine, isolating their actions, practically close to a game, from any moral consideration.

12. The expected military advantage has to be weighed against the risk of civilian casualties.

13. A report by the Royal Society (2017) notes that machines are unable to show "common sense" when the situation becomes complex.

14. The Hague Convention requires that a combatant be "commanded by a person" (International Peace Conference, 1899, Art. 1 of the Annex to the Convention). As the development of autonomous weapons continues, the autonomous defence systems of one State may enter into an interactive relationship with the autonomous weapons of equivalent speed of another State, which may inadvertently lead to the outbreak of an armed conflict before human beings have had the opportunity to react.

15. Already the ability to target at a great distance has opened up the practice of targeted assassinations, which have become easier thanks to robotic systems.

16. Unmanned aerial vehicles equipped with facial recognition software, infrared technology and microphones to record personal conversations constitute an unprecedented violation of the right to privacy.

17. Other examples of this dilemma are proposed by Bonnefon et al $(2016, \mathrm{p} .1576)$ :

- Is it acceptable for an autonomous vehicle, in order to avoid a motorbike, to swerve and enter a wall, the probability of survival in such a case being greater for the passenger of the vehicle than for the driver of the motorbike?

- Should the decision be different when children are in the vehicle, since they have more time to live as future adults and less control over the decision that led them to be in the vehicle?

- If a manufacturer offers different versions of its moral algorithm and a buyer knowingly chooses one, should the buyer be held responsible for the adverse consequences of the algorithm's decisions? 
18. It offers more comfort for the surgeon, a reduction in the length of hospitalisation and less blood loss for the patient, thus minimising the trauma caused, even if they do not necessarily appear to be more efficient than usual surgery and the use of a surgical robot (Kappor, 2014).

19. This acceptance means that the robot must be voluntarily integrated into the life of an elderly person, taking into account the motivation to use a robot, sufficient ease of use and the absence of physical, cognitive and emotional discomfort in the presence of a robot; in addition, its effects on the role of natural carers must be taken into account, so that this acceptance is weighed against its cost, including its maintenance.

20. For example, vacuuming, collecting rubbish, cleaning windows, watering plants, cleaning the pool, ironing, preparing food and drink. Devices such as alarm and security robots, robotic lawnmowers, pet surveillance robots, robotic cradles and robotic shopping assistants are also classified as service robots, as are entertainment robots and, to some extent, companion robots.

21. For example, autonomous vehicles will reduce the number of accidents, surgical robots will increase the precision of medical interventions, service robots will improve the quality of life of the elderly and people with chronic diseases.

22. For example, when an autonomous vehicle causes an accident resulting in human casualties, who should be held responsible? The team of roboticians who designed the vehicle? The manufacturer? The programmer? The seller? The person who decided to buy and use the vehicle? The robot itself?

23. For example, mobile phones allow people to stay in touch, or magnetic resonance imaging (MRI) machines allow doctors to obtain an image of their patients' bodies.

24. https://www.scu.edu/ethics/focus-areas/technolo-gy-ethics/resources/social-robots-ai-and-ethics/

25. Farzaneh and Boyer (2018) put forward the issue of ecology as a possible criterion for choosing between robots and humans in the workplace. From this point of view, the authors suggest making a choice between humans and robots, where possible, based on the comparative cost in terms of energy and raw material consumption between humans and robots.

26. http://www.onrec.com/news/features/the-impor-tance-of-social-media-in-recruiting

27. This law has an important impact on HR professionals because it covers the rights of employees over the personal data that a company keeps: companies can only use the data for the purposes for which it was collected and they cannot collect new data without the employees' permission.

28. https://www.economist.com/democracy-in-ame-rica/2019/05/16/why-san-francisco-banned-the-use-of-facial-recognition-technology

29. https://www.forbes.com/sites/jus-tinwarren/2017/04/06/the-human-point-of-cyber-security/\#15ec13233768

30. For example, ATMs are programmed to give exact change to customers.

\section{REFERENCES}

Alliance des sciences et technologies de numérique. (2016). Éthique de la recherche en robotique. Retrieved from http://cerna-ethics-allistene.org/digitalAssets/38/38704_Avis_robotique_livret.pdf

Alonso, E., Sherman, A.M., Wallington, T.J., Everson, M.P., Field, F.R., Roth, R., \& Kirchain, R.E. (2012). Evaluating rare-earth element availability: A case with revolutionary demand from clean technologies. Environmental Science and Technology, 46, 3406-3414.

Anderson, D., Reimers, K., \& Barretto, C. (2014). Post-Secondary Education Network Security: Results of Addressing the End-User Challenge. INTED2014 (International Technology, Education, and Development Conference).

Anderson, M., \& Anderson, S.L. (2011). General introduction. In M. Anderson \& S.L. Anderson (Eds.), Machine Ethics (pp. 1-4). Cambridge, Cambridge University Press.

Angelo, J.A. (2007). Robotics: A Reference Guide to the New Technology. Westport, Greenwood Press.

Archyworld. (2019). Retrieved from: https://www. archyworldys.com/eric-sadin-artificial-intelligencebreeds-a-progressive-banishment-of-the-human/

Asaro, P.M. (2012). A body to kick, but still no soul to damn: Legal perspectives on robotic. In P. Lin, K. Abney, \& G.A. Bekey (Eds.), Robot Ethics: The Ethical and Social Implications of Robotics (pp. 169-186). London, MIT Press.

Asimov, I. (1942). Círculo vicioso. Analog Science Fiction and Fact.

Asimov, I. (1950). Robot. New York, Gnome Press.

Asimov, I. (1985). Robots and Empire. New York, Doubleday. 
Balding, C. (2016, July 25). Will Robots Ravage the Developing World? Bloomberg View. London, Bloomberg.

Bar-Cohen, Y., \& Hanson, D. (2009). The Coming Robot Revolution: Expectations and Fears About Emerging Intelligent, Humanlike Machines. New York, Springer-Verlag.

Bekey, G.A. (2012). Current trends in robotics: Technology and ethics. In P. Lin, K. Abney, \& G.A. Bekey (Eds.), Robot Ethics: The Ethical and Social Implications of Robotics (pp. 17-34). London, MIT Press.

Beran, T.N., Ramirez-Serrano, A., Kuzyk, R., Fior, M., \& Nugent, S. (2011). Understanding how children understand robots: Perceived animism in child-robot interaction. International Journal HumanComputer Studies, 69(7-8), 539-550.

Bonnefon, J-F., Shariff, A., \& Rahwan, I. (2016). The social dilemma of autonomous vehicles. Science, 352(6293), 1573-1576.

Bruna, M-G., Ducray, L-F., \& Montargot, N. (2017). Décrypter les ambiguïtés de la société post-moderne pour repenser la morphologie de l'entreprise de demain. Une illustration réticulaire. Management \& Sciences Sociales, (23), 64-85.

Bruna, M-G., Montargot, N., \&Peretti, J-M. (2017). Point de vue: Les nouveaux chantiers du management de la diversité. Gestion 2000, 34, 433-462.

Bruna, M-G., Peretti, J-M., \& Yanat, Z. (2016). Les nou-veaux défis de la diversité: Totems et à dépasser et défis à relever. RIMHE, 1(20), 68-88.

Brynjolfsson, E., \& McAfee, A. (2011). Race against the machine: How the digital revolution is accelerating innovation, driving productivity, and irreversibly transforming employment and the economy. Brynjolfsson and McAfee.

Calo, R. (2015). Robotics and the lessons of cyber-law. California Law Review, 103, 513-563.

Carr, N. (2014). The Glass Cage: Automation and Us. New York, W.W. Norton and Company.

Chamayou, G. (2015). A Theory of the Drone. New York, The New Press.

Crandall, J., \& Armitage, J. (2005). Envisioning the Homefront: Militarization, Tracking and Security Culture. Journal of Visual Culture, 4(1), 17-38.

Dhillon, G. (1997). Managing Information System Security. Houndmills, Basingstoke, Hampshire: Macmillan Press LTD.

Driessen, C.P.G., \& Heutinck, L.F.M. (2015). Cows desiring to be milked? Milking robots and the coevolution of ethics and technology on Dutch dairy farms. Agriculture and Human Values, 32(1), 3-20.

Durand, J-P., \& Boyer, R. (2000). L'aprésfordisme. Syros, Paris.

European Commission (EC). (2012). Public Attitudes Towards Robots. Report, Special Eurobarometer 382. Brussels, EC. Retrieved from http://ec.europa.eu/commfrontoffice/publicopinion/archives/ebs/ebs_382_en.pdf

Farzaneh, F., \& Boyer, A. (2018, July). Vacillate between humans and robots in the workplace: The role of eco-logical factors. IRMBAM 2018, pp. 5-7.

Franklin, S. (2014), History, motivations, and core themes. In K. Frankish \& W.M. Ramsey (Eds.), The Cambridge Handbook of Artificial Intelligence (pp. 15-33). Cambridge University Press.

Frey, C.B., \& Osborne, M. (2013). The Future of Employment. Oxford, University of Oxford.

Fukuyama, F. (2002). Our Posthuman Future: Consequences of the Biotechnology Revolution. New York, Farrar, Straus, and Giroux.

Gibilisco, S. (2003). Concise Encyclopedia of Robotics. New York, McGraw-Hill.

Habermas, J. (2003). The Future of Human Nature. Cambridge, Polity Press.

Holder, C., Khurana, V., Harrison, F., \& Jacobs, L. (2016). Robotics and law: Key legal and regulatory implications of the robotics age (part I of II). Computer Law \& Security Review, 32(3), 383-402.

Hottois, G., Missa J-N., \& Perbal, L. (Eds.). (2015). Encyclopédie du trans/posthumanisme. Paris, Vrin.

Ingram, B., Jones, D., Lewis, A., Richards, M., Rich, C., \& Schachterle, L. (2010). A code of ethics for ro-botics engineers. Proceedings of the 5th ACM/IEEE International Conference on HumanRobot Interaction (HRI), pp. 103-104. 
JURI. (2016). Draft Report with recommendations to the to the Commission on Civil Law Rules on Robotics. Committee of Legal Affairs of the European Parliament, Brussels, European Parliament.

Kappor, A. (2014). L'invasion robotique au Canada. Canadian Urology Association Journal, 8, 5-6.

Leenes, R., Palmerini, E., Koops, B-J., Bertolini, A., Salvini, P., \& Lucivero, F. (2017). Regulatory challenges of robotics: Some guidelines for addressing legal and ethical issues. Law, Innovation and Technology, 9(1), 1-44.

Leopold, A. (1949). A Sand County Almanac and Sketches Here and There. New York, Ballantine Books.

Lim, J-S., Chang, S., Maynard, S., \& Ahmad A., (2009). Exploring the Relationship between Organizational Culture and Information Security Culture. 7th Australian Information Security Management Conference. Perth, Western Australia.

Lin, P. (2012). Introduction to robot ethics. In P. Lin, K. Abney, \& G.A. Bekey (Eds.), Robot Ethics: The Ethical and Social Implications of Robotics (pp. 3-16). London, MIT Press.

Matsuzaki, H., \& Lindemann, G. (2015) The autonomy-safety-paradox of service robotics in Europe and Japan: A comparative analysis. IA \& Society, 31(4), 501-517.

Moor, J. (2011). The nature, importance, and difficulty of machine ethics. In M. Anderson \& S.L. Anderson (Eds.), Machine Ethics (pp. 13-20). Cambridge, Cambridge University Press.

Oost, E., \& Reed, D. (2010). Towards a Sociological Understanding of Robots as Companions. In M.H. Lamers \& F.J. Verbeek (Eds.), Human-Robot Personal Relationships. Heidelberg, Berlin, Springer.

Pearson, Y., \& Borenstein, J. (2014). Creating “companions" for children: The ethics of designing esthetic features for robots. IA \& Society, 29, 23-31.

Peláez, L. (2014). The Robotics Divide. A New Frontier in the 21st Century? London, Springer-Verlag.

Reimers, K., \& Andersson, D. (2017). Post-secondary education network security: The end user challenge and evolving threats. ICERI2017 Proceedings (pp. 1787-1796).

Report of COMEST on robotics ethics. (2017). Retrieved from http://www.unesco.org/new/en/so-cialand-human-sciences/themes/comest/robotics-ethics/

Riek, L., \& Howard, D. (2014, April 4). A Code of Ethics for the Human-Robot Interaction Profession. Proceedings of We Robot. Retrieved from https://ssrn.com/abstract=2757805

Rifkin, J. (1995). The End of Work. New York, Putnam.

Rosenberg, J.M. (1986). Dictionary of Artificial Intelligence and Robotics. New York, John Wiley \& Sons.

Rosenberger, R., \& Verbeek, P.P. (2015). A Field Guide to Postphenomenology. In R. Rosenberger \& P.P. Verbeek (Eds.). Postphenomenological Investigations: Essays on Human-Technology Relations (pp. 9-41). London, Lexington Books,

Shelley, M. (1818). Frankenstein ou le Prométhée moderne. Traduction d'Alain Morvan et Marc Porée, Gallimard.

Simut, R.E., Vanderfaeillie, J., Peca A., Van de Perre, G., \& Vanderborght, B. (2016). Children with Autism Spectrum Disorders Make a Fruit Salad with Probo, the Social Robot: An Interaction Study. Journal of Autism Development Disorder, 46, 113-126.

Snell \& Wilmer. (2019). Facing the Issue: San Francisco Bans City Use of Facial Recognition Technology. Retrieved from https://www.jdsupra.com/legalnews/facing-the-issue-san-franciscobans-35144/

Stone, W.L. (2005). The history of robotics. In T.R. Kurfess (Ed.), Robotics and Automation Handbook. Boca Raton, CRC Press.

The Royal Society. (2017). Machine learning: The power and promise of computers that learn by example. London, The Royal Society. Retrieved from https://royalsociety.org//media/policy/projects/ machine-learning/publications/machine-learning-re-port-summary.pdf

Turing, A.M. (1950). Computing Machinery and Intelligence. Mind, 49, 433-460.

Tzafestas, S.G. (2016). Roboethics. A Navigating Overview. Switzerland, Springer. 
Ummat, A., Dubey, A., \& Mavroidis, C. (2004). Bionanorobotics: A field inspired by nature. In Y. BarCohen (Ed.), CRC Handbook on Biomimetics: Mimicking and Inspiration of Biology (pp. 201226). Boca Raton, CRC Press.

Valverdu, J., \& Casacuberta, D. (2009). Handbook of Research on Synthetics Emotions and Sociable Robots: New Applications in Affective Computing and Artificial Intelligence. Hershey, IGI Publishing.

Van de Poel, I. (2016). An Ethical Framework for Evaluating Experimental Technology. Science and Engineering Ethics, 22(3), 667-686.

Verbeek, P.P. (2013). Technology Design as Experimental Ethics. In S. Van den Burg \& Tsj. Swierstra (Eds.), Ethics on the Laboratory Floor (pp. 83-100). Basingstoke, Palgrave Macmillan.

Weir, N.A., Sierra, D.P., \& Jones, J.F. (2005). A Review of Research in the Field of Nanorobotics. SAND2005-6808, Unlimited Release.

Whitby, B. (2011). On computable morality: An examination of machines as moral advisors. In M. Anderson \& S.L. Anderson (Eds.). Machine Ethics (pp. 138-150). Cambridge, Cambridge University Press.

Wise, E. (2005). Robotics Demystified. New York, McGraw-Hill.

Wu, Y-H., Faucounau, V., Boulay, M., Maestrutti, M., \& Rigaud, A.S. (2010). Robotic agents for supporting community-dwelling elderly people with memory complaints: Perceived needs and preferences. Health Informatics Journal, 17(1), 33-40. 